\title{
Initiative populaire fédérale «Pour des soins infirmiers forts»
}

\author{
Helena Zaugga et Yvonne Ribib \\ a Présidente de I'Association suisse des infirmières et infirmiers ASI \\ b Secrétaire générale de I'Association suisse des infirmières et infirmiers ASI
}

Trop peu de professionnels formés, pression temporelle croissante et conditions de travail difficiles nuisent de plus en plus à l'attractivité de la profession infirmière. L'Association suisse des infirmières et infirmiers a lancé en janvier 2017 l'initiative sur les soins infirmiers, une mesure visant à garantir la qualité de la prise en charge infirmière. La population se montre compréhensive face à ces revendications et la FMH soutient l'initiative.

\section{Situation de départ}

Il y a dix ans déjà, on prévoyait que les stratégies de réduction des coûts dans les hôpitaux entraîneraient à long terme une baisse de la qualité, qui, au niveau

\section{Résumé}

L'initiative sur les soins infirmiers a été initiée en raison de l'importante pénurie de personnel résultant de la combinaison entre mesures d'économie et introduction du nouveau système de formation au cours des dix dernières années. C'est surtout au niveau des infirmières diplômées que la pénurie est la plus importante. Aujourd'hui, seuls $43 \%$ des besoins estimés pour cette catégorie professionnelle sont couverts, mais ce chiffre doit augmenter. Pour trois diplômes obtenus en Suisse, deux professionnels venant de l'étranger sont engagés. Le monde politique n'a pas été en mesure de prendre des mesures efficaces contre cette pénurie.

L'initiative sur les soins infirmiers vise, par le biais de revendications de différents ordres et dans l'intérêt des patients et de leur sécurité, à motiver davantage de jeunes à opter pour une profession dans le domaine de la santé et de faire en sorte que les professionnels restent à leurs postes. II s'agit notamment d'augmenter le nombre de diplômes en soins infirmiers par des incitations financières, de définir clairement les rôles professionnels et d'engager toutes les personnes actives dans les soins en fonction de leur formation et de leurs compétences. Le fait de facturer directement les prestations de soins effectuées sous leur propre responsabilité est un moyen de reconnaître les compétences professionnelles des infirmières. Pour accroître l'attractivité de cette profession typiquement féminine, il faut en outre des horaires de travail individualisés et davantage de places pour l'accueil extrafamilial des enfants. Des perspectives de carrière et salariales correspondant aux responsabilités et aux prestations demandées et fournies contribuent à la fidélisation du personnel. du travail sur le terrain, a pour conséquences le renoncement à certains entretiens, des informations lacunaires et un manque de soutien pour la gestion du quotidien [1]. La réforme de la formation entreprise en parallèle (voir encadré, OdASanté 2017) ainsi que la restructuration des offres de formation ont également contribué à la diminution des titres de fin de formation. En 2008 déjà, les besoins en personnel infirmier à venir ne pouvaient être couverts qu'à $50 \%$ [2].

Six ans plus tard, la situation ne s'est que légèrement améliorée. Tandis que le nombre d'assistantes en soins et santé communautaire avec certificat fédéral de capacité (ASSC CFC) formées augmentait pour atteindre $84 \%$ des besoins estimés, celui des infirmières diplômées diminuait à nouveau de $10 \%$ pendant la même période et ne couvre aujourd'hui plus que $43 \%$ des besoins [3]. Conséquence logique du fait que pour trois diplômes indigènes, deux professionnelles étrangères ont été engagées entre 2010 et 2014 [4].

\section{Réaction politique à la pénurie de personnel}

En raison de l'évolution au niveau des professions soignantes, plusieurs parlementaires ont demandé avec insistance que ces professions bénéficient de plus de reconnaissance et que des mesures soient prises pour les rendre plus attractives [5]. Par le biais d'une initiative parlementaire, le conseiller national Joder proposait, concernant le travail infirmier, de faire la distinction entre domaine placé sous leur propre res- 


\section{OdASanté}

OdASanté, Berne, 2017. Cette publication est intégralement protégée par les droits dauteur.

\section{SYSTĖME SUISSE DE FORMATION}

\section{Formation professionnelle supérieure}

Loi fédérale sur la formation professionnelle

\section{Hautes écoles}

Loi fédérale sur l'encouragement des hautes écoles et la coordination dans le domaine suisse des hautes écoles

autres lois féd. sur les hautes écoles

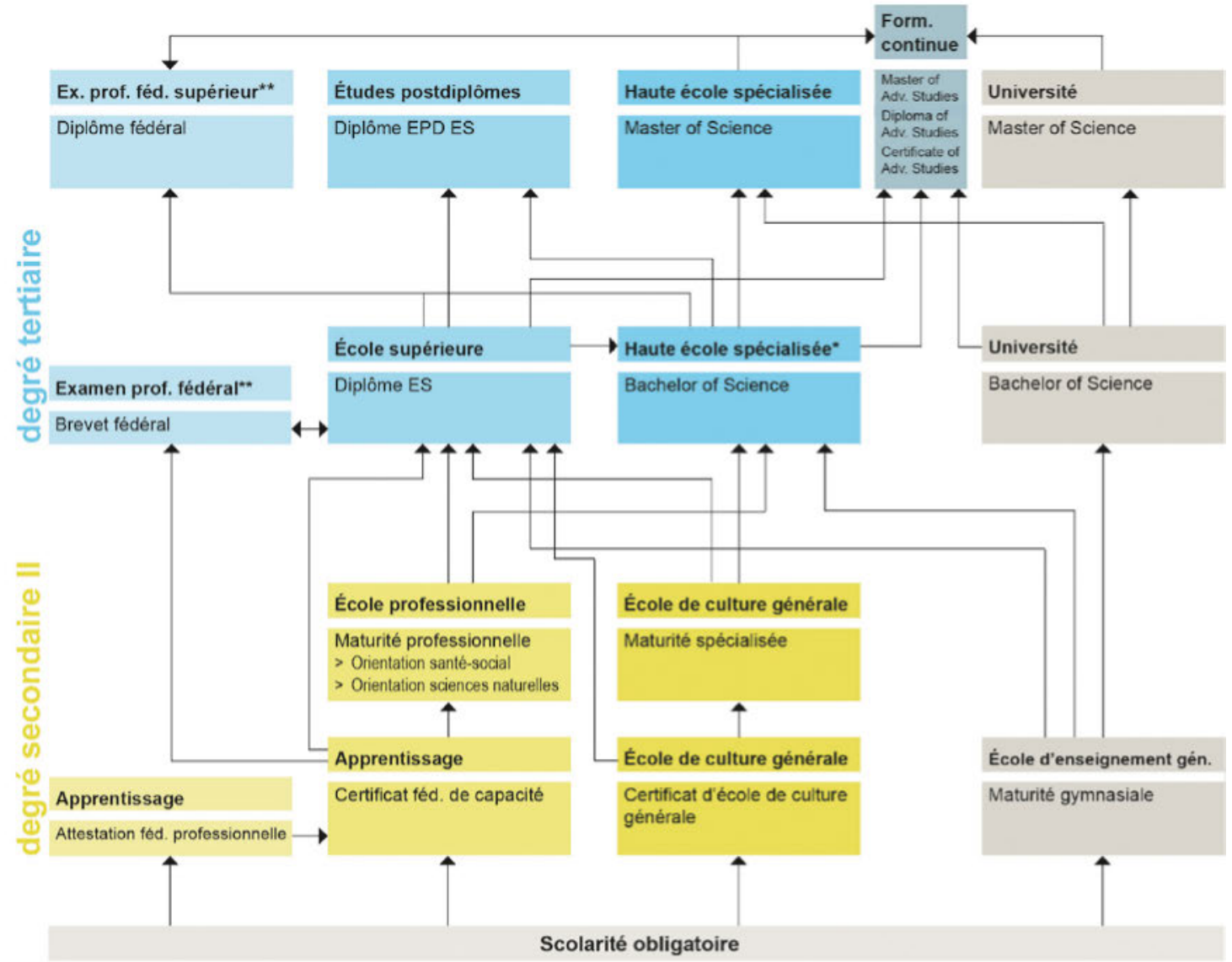

"Sans formation préalable spécifique au domaine des modules complémentaires doivent étre accomplls, c.f. .Profil HES du domaine de la santé du 13.05.04", CDS * Les conditions d'admission sont formulóes dans les réglements d'examen.

OdASanté, Système suisse de formation 2017

ponsabilité et domaine délégué. Dans le domaine placé sous leur propre responsabilité, les infirmières pourraient à l'avenir facturer directement leurs prestations aux caisses-maladie, sans signature d'un médecin. De nombreux politiciens étaient favorables à ce projet de loi, et pourtant le Conseil national a refusé d'entrer en matière sur ce sujet en avril 2016, sans en avoir discuté au préalable.

\section{Pourquoi une initiative populaire}

Avec le Masterplan «Formation aux professions des soins», la Confédération, les cantons et l'OdASanté avaient l'intention d'augmenter le nombre de détenteurs de titres. Cela a réussi pour les CFC. Cependant, il n'est pas possible de cautionner la déclaration officielle annonçant que le nombre d'infermières a augmenté 
grâce au Masterplan [6], bien au contraire: ce nombre a encore diminué. Concrètement, au cours des 5 dernières années, ce sont 10000 diplômes en soins infirmiers de moins qui ont été attribués. Comme cette réalité n’a suscité de l'attention ni au Parlement ni auprès du Conseil fédéral, et qu'aucune mesure efficace n'a été prise pour améliorer la situation, l'ASI a décidé de se tourner vers l'extérieur, avant que les dégâts ne soient plus graves encore. Car le nombre insuffisant d'infirmières diplômées formées en Suisse ne pourra être comblé à long terme par du personnel étranger et la pénurie peut mettre en danger la sécurité des patients. Différentes recherches scientifiques le prouvent: lorsque la part des infirmières diplômées augmente de $10 \%$, le taux de mortalité diminue de quelque 11\% [7].

\section{Le contenu de l'initiative sur les soins infirmiers}

L'objectif de l'initiative est de garantir la couverture en soins dans l'intérêt du patient et de sa sécurité, à l'avenir aussi.

L'initiative demande que l'on investisse dans la formation et la formation continue des infirmières, sachant que le nombre des infirmières diplômées doit augmenter de toute urgence grâce à des incitations adaptées. On parle ici de rôles professionnels clairement définis, de l'engagement de toutes les personnes impliquées dans les soins en fonction, de leur formation et de leurs compétences, de possibilités de carrière et de perspectives salariales correspondant aux responsabilités et aux prestations.

Pour que les infirmières exercent leur activité plus longtemps, les conditions-cadre doivent également devenir plus attrayantes. Pour cette profession typiquement féminine, cela veut dire des conditions favorables à la vie de famille avec des horaires individualisés et plus d'espace pour l'accueil extrafamilial des enfants.

Last but not least, les infirmières et infirmiers devraient pouvoir facturer directement aux caisses- maladie des prestations infirmières typiques comme par exemple l'évaluation des soins requis, les activités de coordination et de conseil de même que des soins de base. Cela contribue à valoriser la profession et à la rendre plus attrayante. A l'heure actuelle déjà, du point de vue juridique, les infirmières doivent assurer leur responsabilité pour ce qui est de l'exécution adéquate de leurs actes. Il s'agit par conséquent de compétences déjà en vigueur dans la pratique.

La récolte de signatures pour l'initiative montre que la population se préoccupe de la couverture en soins en Suisse. L'ASI est confiante et certaine que l'initiative pourra être déposée au cours des mois qui viennent.

\section{Références}

1 Schubert M, Schaffert-Vitfliet B, De Geest S, Aiken L, Sloane DM, Clark S, Abraham I \& Glass T (2005). RICH - Nursing Study. Rationing of Nursing Care in Switzerland $=\mathrm{CH}$. Effects of Rationing of Nursing Care in Switzerland on Patients and Nurses Outcomes. Rapport final Office fédéral de la santé publique.

2 Conférence suisse des directrices et directeurs cantonaux de la santé CDS / Organisation du monde du travail en santé OdASanté (2009). Rapport national sur les besoins en effectifs dans le domaine de la santé. Besoins en personnel et mesures visant à garantir des effectifs suffisants au plan national.

3 Conférence suisse des directrices et directeurs cantonaux de la santé CDS/Organisation du monde du travail en santé OdASanté (2016). Rapport national sur les besoins en effectifs dans le domaine de la santé. Besoins en personnel et mesures visant à garantir des effectifs suffisants au plan national.

4 Merçay C, Burla L, Widmer M (2016). Personnel de santé en suisse -Etat des lieux et perspectives jusqu'en 2030. Obsan, Rapport 71. Neuchâtel, Observatoire suisse de la santé.

5 Heim, Bea. Postulats 10.3127 et 10.3128 du 16.3.2010 «Personnes agées. Garantie des soins» et "Attractivité de la formation en soins infirmiers"; Aubert, Josiane. Interpellation 10.3513 du 17.6.2010 «Formation en soins infirmiers tertiaire A et tertiaire B. Transparence des inscriptions»; Joder, Rudolf. Initiative Parlementaire 11.418 du 16.3.2011 «LAMal. Accorder plus d'autonomie au personnel soignant»; Fraction PDC, Motion 11.3889 du 29.9.2011 «Encourager et soutenir les possibilités de reconversion et les deuxièmes formations pour le personnel de soins».

6 Secrétariat d'Etat à la formation, à la recherche et à l'innovation SEFRI, communiqué de presse du 3.2.2016, https://www. sbfi.admin.ch/sbfi/de/home/aktuell/medienmitteilungen/ news-anzeige-nsb.msg-id-60542.html.

7 Aiken LH, Sloan D, Griffiths P, Rafferty AM, Bruyneel L, McHugh M, Maier CB, Moreno-Casbas T, Ball JE, Ausserhofer D, Sermeus W, in: BMJ Quality \& Safty, Nursing skill mix in European hospitals: cross-sectional study of the association with mortality, patient ratings, and quality of care, November 15, 2016, http://dx.doi. org/10.1136/bmjqs-2016-005567. 